\title{
Glycemic Control and Cardiovascular Outcomes in Diabetes
}

\section{Biswajit Das and Trinath Kumar Mishra*}

Department of Cardiology, SCB Medical College, Odisha, India

\begin{abstract}
While type 1 Diabetes Mellitus (DM) is characterized by insulin deficiency due to pancreatic beta cell destruction, type 2 DM is characterized by a state of long standing insulin resistance (IR), compensatory hyperinsulinemia and varying degrees of elevated plasma glucose (PG), associated with clustering of cardiovascular (CV) risk and development of macrovascular disease prior to diagnosis of DM. Coronary artery disease (CAD) accounts for $70 \%$ of mortality and morbidity in patients with diabetes.
\end{abstract}

Studies made in diabetes care have helped prevent or reduce microvascular complications in type 1 and 2 diabetes. However the same cannot be said about macrovascular disease.

Despite all data concerning the association of diabetes and cardiovascular disease (CVD), the exact mechanism by which diabetes is linked to atherosclerosis is incompletely understood, this is especially true in case of hyperglycemia. The positive effect of intensive glucose management in comparison to non intensive glucose control is far from proven.

DCCT and UKPDS studies have shown that while a glycemic control is important for reaching long term macrovascular complications, early glucose control is far more rewarding (metabolic memory). Later trials like ACCORD, ADVANCE and VADT don't advocate tight glycemic control. In fact, ACCORD trial has shown increased mortality with tight glucose control.

Tight glucose control may be beneficial in selected patients with short disease duration, long life expectancy and no CVD. In critically ill patients a blood glucose target of $140-180 \mathrm{mg} \%$ is fairly reasonable and achievable.

The ESC/EASD guidelines of October 2013,like those of ADA, AHA and ACC continue to endorse a treatment target for glucose control in diabetes of $\mathrm{HbAlc}<7 \%$, based predominantly on microvascular disease with acknowledged uncertainty regarding the effect of the intensive glucose control on CVD risk.

Management of hyperglycemia in diabetics should not be considered in isolation; diabetics require multifactorial intervention for hypertension, dyslipidemia and microalbuminuria besides hyperglycemia. In fact combined use of antihypertensives, aspirin and lipid lowering agent makes it difficult to discern salutary effects of anti hyperglycemic therapy.

Keywords: Diabetes; Glycemic control; Hyperglycemia

\section{Introduction}

Diabetes mellitus (DM) is a condition defined by an elevated level of blood glucose Type 1 diabetes is characterized by deficiency of insulin due to progressive destruction of pancreatic beta cells, progressing to absolute insulin deficiency. Type 2 diabetes is a combination of insulin resistance and beta cell failure in association with obesity and sedentary life style. However, not all overweight/ obese individuals have diabetes and vice- versa.

The increase prevalence of diabetes worldwide has led to a situation where approximately 360 millions people had diabetes in 2011, of which $95 \%$ would have type $2 \mathrm{DM}$ [1]. This number is estimated to increase to 552 million by 2013 and it is presumed that half of these will be unaware of their diabetes status.

The prevalence of diabetes is increasing world wide and more people with diabetes will die or be disabled as a consequence of vascular complications. Prospective studies have shown unambiguous association of blood glucose and glycated hemoglobin level with the risk of major cardiovascular events. In case of subjects with type 1 diabetes, in spite of the fact that CV rate is significantly lower compared with population with type 2 diabetes, their relative risk for coronary heart mortality is 7 fold higher than in matched counterpart without disease.

In spite of all these data concerning the association of diabetes and cardiovascular diseases (CVD), the exact mechanism by which diabetes is linked to atherosclerosis remains incompletely understood. This is especially true in case of hyperglycemia. The role of non-glycemic factors accompanying vast majority of patients with diabetes such as hypertension, dyslipidaemia and hemorrheological abnormality are better understood and appear to be independent of hyperglycemia. There also has been data regarding the future impacts of statins, aspirin, ACE inhibitors and aggressive control of blood pressure on progression of $\mathrm{CV}$ disease. In contrast, the positive effect of intensive glucose management on CV disease outcome is far from proven. Even some studies show a negative influence. The objective of the present article is to analyze trials related to glycemic control in diabetics and assess its impact on CV outcomes.

*Corresponding author: Trinath Kumar Mishra, Professor \& HOD, Department of Cardiology, SCB Medical College, Cuttack- 753007, Odisha, India, Tel: 9437035038; E-mail: drtkmishra@yahoo.com

Received January 16, 2014; Accepted February 10, 2014; Published February 15,2014

Citation: Das B, Mishra TK (2014) Glycemic Control and Cardiovascular Outcomes in Diabetes. J Diabetes Metab 5: 336. doi:10.4172/2155-6156.1000336

Copyright: @ 2014 Das B, et al. This is an open-access article distributed under the terms of the Creative Commons Attribution License, which permits unrestricted use, distribution, and reproduction in any medium, provided the original author and source are credited. 


\section{Glycemic Continuum and CVD}

Type 2 DM develops following a prolonged period of euglycemic insulin resistance(IR) which progresses with development of beta cell failure to frank diabetes with increase risk of vascular complications. While microvascular complications like retinopathy, nephropathy and neuropathy develop with overt hyperglycemia, macrovascular complications like coronary artery disease, cerebrovascular disease and peripheral arterial disease (PAD) appear earlier during the stage of IFG and IGT. Thus these complications are already established when type 2 $\mathrm{DM}$ is diagnosed. Over $60 \%$ pts with type $2 \mathrm{DM}$ develop CVD which is a more severe and costly complication than retinopathy.

\section{Molecular Basis of CVD}

Insulin resistance has an important role in the pathophysiology of diabetes and CVD. Both genetic and environmental factors facilitate its development. The development of CVD in people with IR is characterized by early endothelial dysfunction and vascular inflammation leading to monocyte recruitment, foam cell formation and subsequent development of fatty streaks [1]. Over many years this leads to atherosclerotic plaque which in presence of enhanced inflammation becomes unstable and rupture to promote occlusive thrombus production. Atheroma from people of diabetes has more lipid, inflammatory change and thrombus than those free from DM. These changes occur over a 20-30 year period and are mirrored by the molecular abnormalities seen in untreated insulin resistance and DM.

Type $2 \mathrm{DM}$ patients are obese and the release of free fatty acids (FFA) \& cytokines from adipose tissue directly impairs insulin sensitivity in skeletal muscle and adipose tissue. FFA induces reactive oxygen species production, blunts activation of IRS 1 and P13K - AKT signaling leading to down regulation of insulin responsive GLUT 4 (Figure 1).

Hyperglycemia decreases nitric oxide bioavailability and affects vascular function involving over production of reactive oxygen species [1]. The mitochondrial electron transport chain is one of the first targets of high glucose with a direct increase in super oxide anion formation. A further increase in super oxide anion formation is driven by a vicious cycle involving ROS induced activation of PKC [1]. Mitochondrial ROS in tern activates cascades involved in the pathogenesis of the CV complications including polyol flux, AGE and RAGE. Hyperglycaemia induced ROS generation is involved in the persistence of vascular dysfunction despite normalization of blood glucose levels. This phenomenon is called metabolic memory which explains why vascular complication progresses despite intensive glycemic control. Elevated RoS generation despite euglycemic sensitivity undermines the clinical gold standard of indexing type 2 efficacy by blood glucose status.

Insulin resistant macrophage increases expression of oxidized LDL scavenger receptor-B, promoting foam cell formation and atherosclerosis. Macrophage dysfunction provide a crucial link between diabetes and CVD by both enhancing it and by contributing to the development of fatty streaks and vascular damage.

\section{Impact of Glucose Control on CVD and its Complications}

Randomised controlled trials provide compelling evidence that microvascular complications of DM are reduced by tight glycemic control. However the same cannot be said about macrovascular disease. Several prospective trials have been conducted which have so far failed to provide any conclusive evidence of the superiority of glycemic control in reducing macrovascular complications, or death rates in people with advance disease or those with long duration of diabetes.

\section{Long term effect of glycemic control}

\section{A. Diabetes Control and Complications Trial (DCCT) and Epidemiology of Dibetes Interventions and Complications (EDIC)}

In DCCT the rate of CV events was not significantly altered in the intensive treatment group of patients with type $1 \mathrm{DM} .{ }^{2} \mathrm{After}$ termination of study, $93 \%$ of the cohort were followed for additional 11 years under EDIC, during which the differences in HbAlC disappeared. During the combined 17 years follow up, the risk of any CV event was reduced significantly in the intensive group by $42 \%(9.63 \% \mathrm{p}<0.1)$ [2]

\section{B. United Kingdom Prospective Diabetes Study: (UKPDS)}

C. In the UKPDS trial, 3867 newly diagnosed subjects with type 2 DM were randomised to an intensive glucose control arm involving use of sulfonylurea or insulin and a conventional arm employing life style management. A subgroup of over weight subjects were included in the study that compared intensive glucose control with metformin $(n=343)$ against conventional therapy $(n=411)$. In the insulin and sulfonylurea group, a mean $\mathrm{HbA1C}$ level of $7 \%$ was achieved versus $7.9 \%$ in the control arm over 10 years. Intensive control decreased risk for a composite end point of all diabetes related complications ( $R R R=12 \%, p=0.029)$,and significantly improved microvascular disease risk $(R R R=25 \%, p=0.01)$, where as a trend towards decreased risk of $\mathrm{Ml}$ was observed with intensive control (14.8 $\%$ vs. $16.8 \%, p=0.052$, statistically not significant). Stroke was

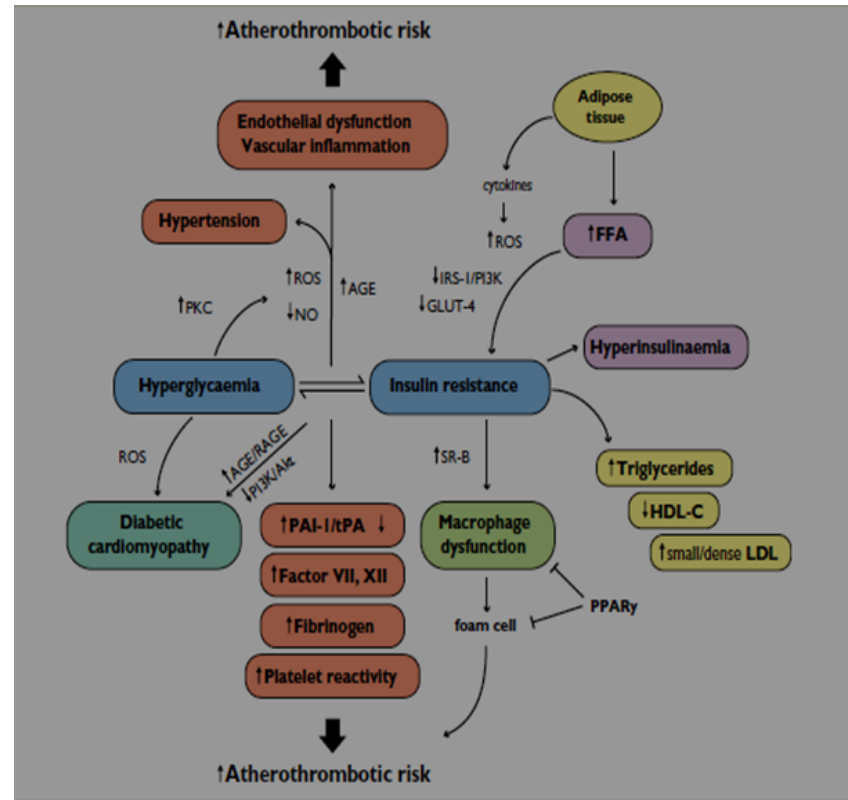

Figure 1: Pathophysiology of Atherosclerosis in Diabetes [1]. AGE: Advanced Glycated End Products; FFA: Free Fatty Acids; GLUT-4: Glucose Transporter 4; NO: Nitric Oxide; PAI-1: Plasminogen Activator Inhibitor 1; PKC: Protein Kinase C; PPARY: Peroxisome Proliferator- Activated Receptor Y; PI3K Phoshphatidylinositide 3- Kinase; RAGE: AGE Receptor; ROS: Reactive Oxygen Species; SR-B: Scavenger Receptor B; tPA: Tissue Plasminogen Activator. 
numerically increased ( $5.6 \%$ vs. $5.2 \%, \mathrm{p}=0.05)$. In over weight subjects, metformin had better glucose control (Alc $>7.4 \%$ vs. $8 \%$ ) as well as significantly improved risk for MI (RRR $=39 \%$, $\mathrm{p}=0.01)$ and for all cause mortality $(\mathrm{RRR}=26 \% \mathrm{p}=0.011)$. In extension phase UKPDS study, the patients were followed up for additional 10 years after completion of the trial, during which difference between HbAlc levels in both the groups disappeared. The follow up showed significantly reduced risk for $\mathrm{MI}$ in those originally randomised to intensive glycemic control both in insulin and sulfonylurea groups (RRR $=15 \%$, $\mathrm{p}-0.01)$ and in the metformin group $(\mathrm{RRR}=33 \%, \mathrm{p}=0.05)$ [3]

There was also significantly $13 \%$ reduction in all cause mortality in the intensively treated group. This persistent benefit generated from early strict glycemic control is known as legacy effect, which outlies the original reduction of $\mathrm{HbAlc}$ and subsequent loss of glycemic control. These observations are similar to those seen in DCCT follow up EDIC study where $\mathrm{CV}$ events, non fatal $\mathrm{MI}$, stroke and $\mathrm{CV}$ death were reduced by $57 \%$ despite loss of glycemic separation [2].

\section{Combined UKPDS and DCCT / EDIC study show that}

1. Glycemic control is important for reducing long term macrovascular complications.

2. Very long follow up period is necessary to demonstrate any benefit.

3. Early glucose control is important (metabolic memory).

\section{Medium term effects of glycemic control}

Action to Control Cardiovascular Risk in Diabetes (ACCORD): The land mark study was designed to determine whether CV disease event rate could be reduced by intensively treating hyperglycemic hypertensive and dyslipidemia in a double $2 \times 2$ factorial design. The trial was based on the hypothesis that a $1.5 \%$ difference in $\mathrm{HbAlc}$ would result in 15\% difference in a population of high risk diabetic individuals having a $3 \%$ annual CVD event rate [4].

The study included 10,251 patients with established type $2 \mathrm{DM}$ and $1 / 3^{\text {rd }}$ having a CV event. Patients were randomised to intensive glucose (targeting $\mathrm{HbAlc}<6 \%$ and achieving a level of $6.4 \%$ ) or standard therapy (targeting HbAlc of $7.0-7.9 \%$ and achieving level of 7.5\%). A variety of glucose lowering therapy was used. There was non significant trend towards reduction in primary outcome of trial (a composite of non fatal $\mathrm{Ml}$, stroke or $\mathrm{CV}$ death) with intensive control. However, unexpectedly there was higher all cause mortality (CR-1.22, $95 \% \mathrm{Cl}$ $1.01-1.46, \mathrm{p}=0.04)$. Higher rate of severe hypoglycaemia and weight gain were reported in intensive glycemic control group. Patients with high $\mathrm{HbAlc}$ level at base line were at higher rate of hypoglycaemia as were those who did not respond properly with a fall of HbAlc in intensive control group. The explanation for incremental mortality remains unresolved: possible explanations include hypoglycaemia precipitating $\mathrm{CV}$ death, pernicious effects of specific drug or combinations and a chance finding.

Advance trial: The study was conducted to determine whether intensive lowering would reduce risk of microvascular and macrovascular events in individuals with type $2 \mathrm{DM}$ and vascular risk factors compared to standard conventional case. The study involved 11,140 subjects. The mean duration of follow up was 5 years. The patients were randomised to intensive versus standard glucose control with gliclazide plus other drugs in the intensive arm compared with other drugs in the standard control group. Mean HbAlc achieved was $6.5 \%$ in the intensive group compared with $7 \%$ in the standard group. The incidence of combined major macrovascular and microvascular events was significantly reduced (HR-0.9, 95\% CI $0.82-0.98, \mathrm{p}=0.01$ ) in the intensive control group. This was primarily driven by reduction in progression of albuminuria or emergence of new nephropathy. The $\mathrm{CV}$ component of the primary event was not significantly reduced by intensive glucose control. There was no evidence of increase in all cause mortality. Actually there is a non significant trend towards reduction in all cause mortality [5].

The VADT study: The trial included American veterans, and $90 \%$ were males. A variety of glucose lowering agents was used including metformin, glimepiride, rosiglitazone and insulin. An HbAlc of $6.9 \%$ was achieved in the intensified control arm compared with $\mathrm{HbA1c}$ of $8.4 \%$ in the standard treatment arm. After a median follow up of 6.5 years, no significant lowering of composite CV outcomes was noted in the intensive control group. Severe hypoglycemia was more prevalent in the intensive control group. Benefits of intensive control were apparent only in individuals with shorter duration of diabetes, lower HbAlc and absence of CVD at base line. Table 1 shows the baseline characteristics of ACCORD, ADVANCE \& VADT trials [6].

\section{Insights from ACCORD, ADVANCE and VADT Trial}

- Important finding of all 3 studies is the suggestion that a beneficial effect of glycemic control intervention is more likely in association with less disease duration.

- In the ACCORD study participants with base line A1c < $8 \%$ rather than having adverse effects of intensive glycemic treatment on mortality, showed a significant reduction in primary out come favoring such treatment. Similarly in ADVANCE trial, the combined macro and microvascular primary outcome benefit of glycemic control intervention was seen in participants without a baseline history of macrovascular disease. Similarly in the VADT trial, patients who had composite out come event had longer diabetes duration, higher $\mathrm{HbAlc}$ and coronary arterial calcification.

- Effect of hypoglycaemia may be of importance. In the ACCORD study, although investigators stated that this was not a mediator of increased mortality associated with intensive therapy, intensive interventions was associated with significant severe hypoglycaemia. The ADVANCE and VADT study group similarly have reported high incidence of severe hypoglycaemia.

- A meta analysis of these 3 trials suggest that $\mathrm{HbA1c}$ reduction of $1 \%$ is associated with $15 \%$ of relative risk reduction in non fatal Ml, but without benefit on stroke or all cause mortality.

- Conclusion from these 3 trials is that intensive glycemic control should be appropriately applied in an individualized manner taking into account age, duration of diabetes and history of CVD.

- Despite the fact that ACCORD, ADVANCE and VADT showed no benefit of intensive glucose control on primary CV endpoints in Type $2 \mathrm{DM}$, subgroup analyses suggest that any potential benefit on CV outcomes and mortality depends upon multiple interrelated factors such that medications capable of exerting direct $\mathrm{CV}$ therapeutic effects may be required to see a CV benefit. 
- One should also remember that HbAlc cutoff makes less sense for the cardiac events because cardiovascular risk depends upon various strong risk factors like hypertension and smoking [7] (Table 1).

\section{Glucose Control in ACS}

Elevated plasma glucose during an ACS is associated with a serious prognosis in patients with DM than without diabetes. Hyperglycemia may relate to previously undetected glucose perturbations but also to stress induced catecholamine release increasing FFA concentration, decreased insulin production and increasing insulin resistance and glycogenolysis with a negative impact on myocardial metabolism and function.

Two strategies have been tasted in an attempt to improve prognosis in patients with ACS

\section{Metabolic modulation}

Metabolic modulation by means of glucose-insulin-potassium infusion regardless of presence of DM or elevated PG, is based on the assumption that increase in intracellular potassium stabilizes the cardiac myocytes and facilitates glucose transport into the cell. Other potential benefits include decreased production of FFA, improved use of glucose for energy production and improved endothelial function and fibrinolysis. Despite these proposed mechanistic benefits of glucose, potassium and insulin therapy, the strategy has been proven futile in CREATE trial which enrolled more than 20000 patients with $\mathrm{Ml}$ who randomised to G\&K therapy versus usual care. No benefit of G\&K therapy was demonstrated. This lack of effect may be due to increased PG or negative effect of fluid load induced by G\&K infusion.

The DIGAMI trial, which is often misinterpreted as a trial of intensive glucose control is actually a glucose insulin infusion therapy trial [8]. The first DIGAMI trial randomised 620 patients with DM and AMI to $>24 \mathrm{hrs}$ insulin-glucose infusions followed by multidose insulin, or routine glucose lowering therapy. Mortality after 3-4 yrs was significantly reduced in the intervention group [8]. However DIGAMI-2 failed to replicate this prognostic benefit. The plausible reason for this discrepancy was that in DIGAMI-l admission HbAlc decreased more (1.5\%) from a higher level $(9.1 \%)$ compared with $0.5 \%$ from $8.3 \%$ in DIGAMI-2. Since DIGAMI-2 trial did not achieve a difference in glucose control between intensively treated and control groups, it is still an open question as to whether glucose lowering is beneficial.

\section{Glucose control in ICU setting}

In 2001 Van den Berghe published a randomised controlled trial of critically ill surgical pts showing that tight glucose control reduced hospital mortality [9]. Since the greatest decrease in death occurred in subgroup of pts with multi system organ failure, it was speculated that benefits of tight glucose control might extend to medical ICU patients as well. However subsequent trials by the same group couldn't demonstrate any benefit with tight glycemic control. Further recent trial like VISEP and European glucontrol showed trend for increased rate with tight glucose control. The NICE SUGAR trial in fact demonstrated an actual $14 \%$ increase in mortality rate with intensive glucose regimen [10].

Few of these trials assessing glucose control in ICU settings included ACS patients. Therefore, general applicability of the observation remains uncertain. Because of paucity of data on tight glycemic control a glucose target of $<180 \mathrm{mg} \%$ is a reasonable approach in ACS pts.

\section{Why Lower is not Necessarily Better?}

The UKPDS study was the first to provide evidence that in newly diagnosed type $2 \mathrm{DM}$ patients intensive glucose control may reduce the risk of microvascular complications, also with modest effect on CV outcomes. Thus the concept 'the lower, the better' (glucose level) was proposed by all diabetology guidelines as a paradigm for type $2 \mathrm{DM}$ patients. However, this concept has been challenged by 3 landmark trials: ACCORD, ADVANCE and VADT.

Numerous potential reasons have been put forth to explain the lack of benefits with intensive glucose control therapy. These include pernicious effects of specific drugs or drug combinations, increased incidence of hypoglycaemia precipitating CV death and a mere chance finding. The current glycemic target is $<7 \%$ of $\mathrm{HbAlc}$ with individualization of therapy (Table 2).

\section{Hypoglycemia and Adverse CV Events}

In the ACCORD trial, which included diabetic patients with $\mathrm{CV}$

\begin{tabular}{|c|c|c|c|c|c|c|}
\hline & \multicolumn{2}{|l|}{ ACCORD } & \multicolumn{2}{|c|}{ ADVANCE } & \multicolumn{2}{|l|}{ VADT } \\
\hline $\mathrm{N}$ & \multicolumn{2}{|l|}{10,251} & \multicolumn{2}{|l|}{11,140} & \multicolumn{2}{|l|}{1791} \\
\hline Age (mean, years) & \multicolumn{2}{|l|}{62} & \multicolumn{2}{|l|}{66} & \multicolumn{2}{|l|}{60} \\
\hline BMI (mean, kg/m²) & \multicolumn{2}{|l|}{32} & \multicolumn{2}{|l|}{28} & \multicolumn{2}{|l|}{31} \\
\hline Follow-up (mean, years) & \multicolumn{2}{|l|}{3.5} & \multicolumn{2}{|l|}{5} & \multicolumn{2}{|l|}{5.6} \\
\hline A1c target & \multicolumn{2}{|c|}{$<6.0 \%$ versus $7.0 \%-7.9 \%$} & \multicolumn{2}{|c|}{$\leq 6.5 \%$ versus "standard" } & \multicolumn{2}{|c|}{$<6 \%$ versus $8 \%-9 \%$} \\
\hline Baseline A1c (mean) & \multicolumn{2}{|l|}{$8.3 \%$} & \multicolumn{2}{|l|}{$7.5 \%$} & \multicolumn{2}{|l|}{$9.4 \%$} \\
\hline Endpoint A1c (mean) & $\begin{array}{l}\text { Intensive } \\
6.4 \%\end{array}$ & $\begin{array}{l}\text { Standard } \\
7.5 \%\end{array}$ & $\begin{array}{l}\text { Intensive } \\
6.43 \%\end{array}$ & $\begin{array}{l}\text { Standard } \\
7.0 \%\end{array}$ & $\begin{array}{l}\text { Intensive } \\
6.9 \%\end{array}$ & $\begin{array}{l}\text { Standard } \\
8.4 \%\end{array}$ \\
\hline Severe hypoglycemic events & $\begin{array}{l}\text { Intensive } \\
10.5 \%\end{array}$ & $\begin{array}{l}\text { Standard } \\
3.5 \%\end{array}$ & $\begin{array}{l}\text { Intensive } \\
2.7 \%\end{array}$ & $\begin{array}{l}\text { Standard } \\
1.5 \%\end{array}$ & $\begin{array}{l}\text { Intensive } \\
8.5 \%\end{array}$ & $\begin{array}{l}\text { Standard } \\
2.1 \%\end{array}$ \\
\hline Weight change & $\begin{array}{l}\text { Intensive } \\
+3.5 \mathrm{~kg}\end{array}$ & $\begin{array}{l}\text { Standard } \\
+0.4 \mathrm{~kg}\end{array}$ & $\begin{array}{l}\text { Intensive } \\
-0.1 \mathrm{~kg}\end{array}$ & $\begin{array}{l}\text { Standard } \\
-1.0 \mathrm{~kg}\end{array}$ & $\begin{array}{l}\text { Intensive } \\
+8.1 \%\end{array}$ & $\begin{array}{l}\text { Standard } \\
+4.1 \%\end{array}$ \\
\hline Major macrovascular or microvascular event & \multicolumn{2}{|c|}{ Not reported } & \multicolumn{2}{|c|}{$0.9(0.82-0.98), P=0.01$} & \multicolumn{2}{|c|}{$0.88(0.74-1.05), P=0.14$} \\
\hline Nonfatal MI/stroke, CV death & \multicolumn{2}{|c|}{ HR $0.9(0.78-1.04), P=0.16$} & \multicolumn{2}{|c|}{$0.94(0.84-1.06), P=0.32$} & \multicolumn{2}{|c|}{ Not reported } \\
\hline All-cause mortality & \multicolumn{2}{|c|}{ HR $1.22(1.01-1.46), P=0.04$} & \multicolumn{2}{|c|}{$0.93(0.83-1.06), P=0.28$} & \multicolumn{2}{|c|}{$1.07(0.81-1.42), P=0.62$} \\
\hline Nonfatal MI & \multicolumn{2}{|c|}{ HR $0.76(0.62-0.92), P=0.004$} & \multicolumn{2}{|c|}{$0.98(0.77-1.22), P=\mathrm{NS}$} & \multicolumn{2}{|c|}{$0.82(059-1.14), P=0.24$} \\
\hline
\end{tabular}

ACCORD: Action to Control Cardiovascular Risk in Diabetes trial; ADVANCE: Action in Diabetes and Vascular Disease: preterAx and diamicro N-MR Controlled Evaluation trial; A1c: Gglycosylated Hemoglobin; BMI: Body Mass Index; CV: Cardiovascular; MI: Myocardial Infarction; VADT: Veterans Affairs Diabetes Trial

Table 1: Baseline characteristics of ACCORD, ADVANCE \& VADT trials. 
While an $\mathrm{HbA} 1 \mathrm{c}$ target of less than $7 \%$ to reduce microvascular disease is a generally accepted level, the evidence for an $\mathrm{HbA} 1 \mathrm{c}$ target in relation to macrovascular risk is less compelling

Consensus indicates that an $\mathrm{HbA} 1 \mathrm{c}$ of less than $7 \%$ should be targeted but with acknowledgement of need to pay attention to the individual requirement of the patient. Fasting plasma glucose should be less than $120 \mathrm{mg} \%(7.2 \mathrm{mmol} / \mathrm{l})$ and postprandial less than $160-180 \mathrm{mg} \%(9-10 \mathrm{mmol} / \mathrm{l})$ on an individualized basis. Ideally tight glycemic control should be started early in the course of the disease in younger people and without attendant comorbidities Stringent targets like HbAlc 6-6.5\% may be considered in selected patients with short disease duration, long life expectancy \& no CVD, if it can be achieved without hypoglycaemia or other adverse effect.

For critically ill indoor patients insulin therapy is indicated at a threshold of no greater than $180 \mathrm{mg} \%$ ( IOmmol/l) (ADA2008)

Once insulin therapy has been started in critically ill patients a glucose range of $140-180 \mathrm{mg} \%$ is recommended.

With the preferred method of intravenous insulin infusion, frequent glucose monitoring is essential to minimize occurrence of hypoglycaemia and to achieve optimal glucose control.

Tight glucose control $(80-110 \mathrm{mg} \%)$ has not been associated with mortality benefit in many trials. In past some trials show increase mortality.

Table 2: Current Glycemic Targets (ESC/EASD guidelines) [1].

disease or high CV risk, symptomatic, severe hypoglycaemia was associated with higher mortality in patients in both study arms [4]. ADVANCE trial also showed that occurrence of severe hypoglycemic episodes has a detrimental effect on CV outcome.

The ORIGIN trial also showed evidence corroborating hypoglycaemia with adverse CV outcomes [11]. The trial randomised 12,537 people at high risk of CVD plus IGF, IGT or type 2DM to receive insulin glargine (with a target FBS level of $<95 \mathrm{mg} \%$ ) versus standard care. After a median follow up of 6.2 years, the rates of incident CV outcome were similar in both the groups. In this population of ORIGIN trial, severe hypoglycaemia occurred in $5.7 \%$ \& $1.8 \%$ patients assigned for insulin glargine and standard therapy groups respectively. Severe hypoglycaemia was associated with a greater risk for primary outcome, mortality, CV deaths and arrhythmic deaths.

Compensatory mechanisms induced by hypoglycaemia, such as enhanced catecholamine release, may aggravate myocardial ischemia and provoke arrhythmia. Still then, it remains unclear weather hypoglycaemia is simply a marker of disease severity or contributes to adverse outcomes. Hypoglycemic episodes probably identify patients at risk for other reasons like malnutrition, $\mathrm{HF}$, and renal dysfunction.

\section{Cardiovascular Effects of Drugs used in Diabetes}

Few data are available regarding the net cardiovascular safety and efficacy of medications used to control glucose level in diabetes.

Metformin has best track record of safety, tolerability and low hypoglycemia risk. This drug remains the drug of first choice.

Concern always exists regarding ability of sulfonylurea, to impair ischaemic preconditioning. However, UKPDS has been able to allay such fear to some extent.

Of thiazolidinediones, rosiglitazone was withdrawn from market because of fear of increased myocardial infarction risk. Recently, it has been reintroduced. Pioglitazone reduces myocardial infarction risk but can cause fluid retention.

Dipeptidyl peptidase 4 inhibitors have so far shown to have no adverse cardiovascular outcomes. Their safety track appears good.

Insulin increases the risk of hypoglycemia and retrospective studies show adverse outcome when insulin is used in diabetics with heart failure.

\section{Conclusions}

As the disease diabetes assumes alarming proportions and threatens to become the modern pandemic, every effort should be made to prevent diabetes related cardiovascular complications. Interventions to reduce fasting blood glucose levels have unfortunately been not translated to better cardiovascular outcomes in all individuals. Recent trials like ACCORD, ADVANCE, and VADT challenge this proposition. However, metaanalysis of these trials suggests that the subgroups of diabetics with shorter duration of illness are beneficial from tight glycemic control $(\mathrm{HbA} 1 \mathrm{c}<7 \%)$. Hypoglycemia is always an issue, when the physician aims for tight glycemic control. As hypoglycemia adversely affects cardiovascular homeostasis, every effort should be made to avoid it at all costs while going for tight glycemic control.

\section{References}

1. Authors/Task Force Members, Rydén L, Grant PJ, Anker SD, Berne C, et al (2013) ESC Guidelines on diabetes, pre-diabetes, and cardiovascular diseases developed in collaboration with the EASD: the Task Force on diabetes, prediabetes, and cardiovascular diseases of the European Society of Cardiology (ESC) and developed in collaboration with the European Association for the Study of Diabetes (EASD). Eur Heart J 34: 3035-3087.

2. (1993) The effect of intensive treatment of diabetes on the development and progression of long-term complications in insulin-dependent diabetes mellitus. The Diabetes Control and Complications Trial Research Group. N Engl J Med 329: 977-986.

3. Holman RR, Paul SK, Bethel MA, Matthews DR, Neil HA (2008) 10-year followup of intensive glucose control in type 2 diabetes. N Engl J Med 359: 1577 1589.

4. Action to Control Cardiovascular Risk in Diabetes Study Group, Gerstein HC Miller ME, Byington RP, Goff DC Jr, et al. (2008) Effects of intensive glucose lowering in type 2 diabetes. N Engl J Med 358: 2545-2559.

5. ADVANCE Collaborative Group, Patel A, MacMahon S, Chalmers J, Neal B et al. (2008) Intensive blood glucose control and vascular outcomes in patients with type 2 diabetes. N Engl J Med 358: 2560-2572.

6. Duckworth W, Abraira C, Moritz T, Reda D, Emanuele N, et al. (2009) Glucose control and vascular complications in veterans with type 2 diabetes. $\mathrm{N}$ Engl J Med 360: 129-139.

7. Kowall B, Rathmann W (2013) HbA1c for diagnosis of type 2 diabetes. Is there an optimal cut point to assess high risk of diabetes complications, and how well does the 6.5\% cutoff perform? Diabetes Metab Syndr Obes 6: 477-491.

8. Malmberg K, Ryden L, Efendic S, Herlitz J, Nicol P, et al. (1995) Randomised trial of insulin glucose infusion followed by subcutaneous insulin treatment in diabetic patients with acute myocardial infarction(DIGAMI Study);Effects on mortality at 1 year. J Am Coll Cardiol 26: 57-65.

9. van den Berghe $G$, Wouters $P$, Weekers $F$, Verwaest $C$, Bruyninckx F, et al (2001) Intensive insulin therapy in critically ill patients. N Engl J Med 345: 13591367.

10. NICE-SUGAR Study Investigators, Finfer S, Chittock DR, Su SY, Blair D, et al (2009) Intensive versus conventional glucose control in critically ill patients. N Engl J Med 360: 1283-1297.

11. ORIGIN Trial Investigators, Gerstein HC, Bosch J, Dagenais GR, Díaz R, et al. (2012) Basal insulin and cardiovascular and other outcomes in dysglycemia. N Engl J Med 367: 319-328. 